\section{Kidney \\ Blood Pressure Research}

\title{
Interferon (IFN)-Induced Protein 35 (IFI35), a Type I Interferon-Dependent Transcript, Upregulates Inflammatory Signaling Pathways by Activating Toll-Like Receptor 3 in Human Mesangial Cells
}

\author{
Tadaatsu Imaizumi ${ }^{a}$ Chikashi Yano ${ }^{a}$ Akiko Numata ${ }^{a}$ Koji Tsugawab \\ Ryo Hayakaria Tomoh Matsumiya ${ }^{a}$ Hidemi Yoshida ${ }^{a}$ Shojiro Watanabe ${ }^{b}$ \\ Kazushi Tsurugab Shogo Kawaguchic Manabu Murakamid Hiroshi Tanakab,e \\ aDepartment of Vascular Biology, bepartment of Pediatrics, 'Department of Gastroenterology and \\ Hematology, dDepartment of Pharmacology, Hirosaki University Graduate School of Medicine, Hirosaki; \\ eDepartment of School Health Science, Hirosaki University Faculty of Education, Hirosaki, Japan
}

\section{Key Words}

$\mathrm{CCL} 5 \cdot \mathrm{CXCL10} \cdot \mathrm{IFI} 35 \cdot \mathrm{IFN}-\beta \cdot \mathrm{MDA} 5 \cdot$ Mesangial cells $\bullet$ Poly IC $・$ TLR3

\begin{abstract}
Background/Aims: Activation of Toll-like receptor 3 (TLR3) signaling followed by type I interferon (IFN) expression is crucial in antiviral and "pseudoviral" immune reactions in renal mesangial cells (MCs). These reactions are probably involved in the pathogenesis of chronic kidney disease (CKD). However, the role of IFN-induced 35-kDa protein 35 (IFI35), a type I IFNdependent transcript, in glomerular inflammation is unclear. Here, we aimed to investigate the expression and the role of IFI35 in IFN- $\beta /$ retinoic acid-inducible gene-I (RIG-I)/CCL5 and IFN- $\beta$ /melanoma differentiation-associated gene 5 (MDA5)/CXCL10 axes in MCs. Methods: We treated human MCs with polyinosinic-polycytidylic acid (poly IC), an authentic doublestranded RNA, then analysed the IFI35 expression by reverse transcription-polymerase chain reaction and western blotting. To examine the regulation of IFI35 expression, we subjected MCs to RNA interference (siRNA) against IFN- $\beta$, RIG-I, and MDA5. Results: Activation of TLR3 by poly IC induces the IFI35 expression in MCs. siRNA against IFN- $\beta$ inhibited poly IC-induced IFI35 expression. Knockdown of IFI35 resulted in a decrease of poly IC-induced RIG-I and MDA5 protein as well as decreased CCL5 and CXCL10 mRNA and protein expression. However, it did not affect the expression of none of phosphorylated signal transducers or activator of transcription (STAT) 1 protein, or RIG-I and MDA5 in mRNA levels. Conclusion: Regional expression of IFI35 and its dysregulation may be involved in the pathogenesis of glomerular inflammation in CKD.




\section{Kidney Blood Pressure Research}

\section{Introduction}

Expression of mesangial proinflammatory chemokines and cytokines during innate immunity plays a pivotal role in the pathogenesis of chronic kidney disease (CKD) pathogenesis [1-4]. Non-infectious stimuli, such as endogenous ligands, in addition to viral infections can activate Toll-like receptor (TLR) 3 signaling pathways in mesangial cells (MCs). The inflammatory processes induced by these pathways affect residual glomerular cells $[3,4]$. Because MCs have been reported to produce a wide variety of proinflammatory chemokines and cytokines that play roles in regional immune and inflammatory reactions in the kidney, MCs themselves are crucial in CKD pathogenesis [5]. We previously studied TLR3 signaling cascades triggered by polyinosinic-polycytidylic acid (poly IC), a synthetic analogue of viral dsRNA, in cultured normal human MCs, and found that treatment of cells with poly IC followed by interferon (IFN)- $\beta$ expression induces the phosphorylation of the signal transducers and activators of transcription 1 (STAT1) protein [6]. IFN-stimulated genes (ISGs) are then induced and they modulate various biological reactions [6-9]. Further, we found that cytosolic RNA recognition sensors, retinoic and-inducible gene-I (RIG-I) and melanoma differentiation-associated gene 5 (MDA5), are also involved in the TLR3 activation-related proinflammatory signaling and the downstream expressions of CCL5 and CXCL10 (TLR3/IFN- $\beta$ /RIG-I/CCL5 and TLR3/IFN- $\beta$ /MDA5/CXCL10 pathways) in MCs $[10,11]$. IFN-induced 35-kDa protein (IFI35; also named as IFP35) is one of ISGs [12]. IFI35 molecule has a leucine zipper domain in its structure and is thought to regulate the gene expression, although it lacks the DNA-binding motifs [12]. It was recently reported that IFI35 negatively regulates antiviral signaling of RIG-I in HEK293 cells infected with Sendai virus [13]. Furthermore, IFI35 expression has been reported in fibroblasts, macrophages, epithelial cells and HeLa cells [12]. However, there have been no reports on the expression and function of IFI35 in MCs. Therefore, we investigated the expression and the role of IFI35

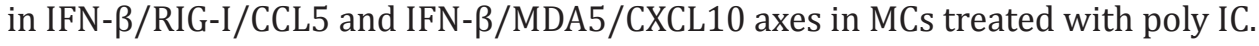

\section{Materials and Methods}

\section{Reagents}

Poly IC and anti-actin rabbit IgG were purchased from Sigma (St Louis, MA, USA). Small interfering RNAs (siRNAs) against IFN- $\beta$ and an anti-RIG-I antibody were previously described [14]. siRNAs against IFI35 (SI00445767), RIG-I (SI02660777), MDA5 (SI03649037), and the non-silencing negative control siRNA (1027281) were purchased from Qiagen (Hilden, Germany). Lipofectamine RNAiMAX was purchased from Invitrogen (Frederick, MD, USA). The illustra RNAspin kit was purchased from GE Healthcare (Buckinghamshire, UK). The dNTP mix was purchased from Thermo Fisher Scientific (Asheville, MA, USA). SsoAdvanced Universal SYBR Green Supermix was purchased from Bio-Rad (Hercules, CA, USA). Oligonucleotide primers for polymerase chain reaction (PCR) were synthesized by Greiner Japan (Atsugi, Japan). Precast polyacrylamide gels were purchased from ATTO (Tokyo, Japan). The anti-IFI35 mouse IgG (sc-100769), anti-phosphorylated STAT1 mouse IgG (sc-136229) and anti-STAT1 rabbit IgG (sc-592) antibodies were purchased from Santa Cruz Biotechnology (Dallas, TX, USA). The anti-MDA5 antibody was purchased from Immuno-Biological Laboratories (Takasaki, Japan). Enzyme-linked immunosorbent assay (ELISA) kits for CCL5 and CXCL10 were purchased from R\&D systems (Minneapolis, MN, USA).

Cells

Normal human MCs were purchased from Lonza (Walkersville, MD, USA), and were cultured using mesangial medium according to the supplier's protocol as previously described [6-11]. The cells were treated with 2 to $50 \mu \mathrm{g} / \mathrm{mL}$ poly IC, and were incubated for up to $24 \mathrm{~h}$. In experiments to examine the effect of RNA interference against IFN- $\beta$, IFI35, RIG-I or MDA5, the cells were transfected with specific siRNA or with a non-silencing negative control using Lipofectamine RNAiMAX reagent according to the manufacturer's protocol. After $48 \mathrm{~h}$ incubation, the cells were subsequently treated with $30 \mu \mathrm{g} / \mathrm{mL}$ poly IC. The cells were subjected to quantitative real-time reverse transcription-PCR (RT-PCR) or western blot analysis after 


\section{Kidney \\ Blood Pressure Research}

Kidney Blood Press Res 2016;41:635-642

\begin{tabular}{|l|l|}
\hline DOI: 10.1159/000447932 & ( 2016 The Author(s). Published by S. Karger AG, Basel
\end{tabular}

Published online: September 17, 2016 www.karger.com/kbr

Imaizumi et al.: IFI35 and TLR3 Signaling Pathways in Hman MCs additional incubation as indicated. Cell viability was not affected under the conditions used.

RNA extraction and quantitative RT-PCR analysis

Total RNA was extracted from cells after incubation and single-strand cDNA was generated using a reverse transcriptase. The cDNA for IFI35, IFN- $\beta$, RIG-I, MDA5, CCL5, CXCL10 or 18S ribosomal RNA was amplified using SsoAdvanced Universal SYBR Green Supermix. The primers used are summarized in Table 1.

\section{Western blot analysis}

After incubation, the cells were lysed using Laemmli's reducing sample buffer. Cell lysates were subjected to electrophoresis on a $7.5 \%$ or $12.5 \%$ polyacrylamide gel, and proteins were transferred to polyvinylidene difluoride membranes. The membrane was incubated with an antibody against IFI35 (1:1000 dilution), phosphorylated STAT1 (P-STAT1, 1:5000), STAT1 (1:10000), RIG-I (1:10000), MDA5 (1:1000) or actin (1:5000 dilution), followed by incubation with a horseradish peroxidaselabelled secondary antibody. Immunodetection was performed using a chemiluminescence substrate.

\section{ELISA for CCL5 and CXCL10}

Cell-conditioned medium was collected after incubation and was briefly centrifuged. The concentration of CCL5 and CXCL10 in the supernatant was determined using ELISA kits according to the manufacturer's protocol.

Statistical Analysis

Statistical significance was evaluated using a Student $t$-test, and $\mathrm{p}<0.01$ was considered to be significant.

\section{Results}

Expression of IFI35 is induced by poly IC in cultured normal human mesangial cells

Treatment of cultured normal human MCs with poly IC resulted in the induction of IFI35 mRNA and protein in a concentration- (Fig. $1 \mathrm{~A}$ and B) and time- (Fig. 1C and D) dependent manner. The expression of IFI35 protein reached a maximum 6-24 h post treatment with poly IC (Fig. 1D).

IFN- $\beta$ is involved in the poly IC-induced expression of IFI35

Transfection of MCs with siRNA against IFN- $\beta$ significantly suppressed the expression of IFI35 mRNA (Fig. 2A) and protein (Fig. 2B) induced by poly IC treatment. However, knockdown of neither RIG-I nor MDA5 affected the poly IC-induced expression of IFI35 (Fig. $2 \mathrm{~A}$ and $\mathrm{B})$.

Knockdown of IFI35 decreased the protein expression of RIG-I, MDA5, CCL5 and CXCL10, and $m R N A$ s expression of CCL5 and CXCL10

Transfection of MCs with siRNA against IFI35 markedly inhibited the IFI35 expression induced by poly IC (Fig. 3B). Knockdown of IFI35 decreased the protein expression of RIG-I and MDA5 induced by poly IC, but did not affect protein expression of STAT1 or P-STAT1 


\section{Kidney \\ Blood Pressure Research}

Fig. 1. Treatment of cultured normal human mesangial cells with poly IC induced the expression of IFI35 in a concentration- and time-dependent manner. RNA was extracted from cultured normal human MCs after poly IC treatment at various concentrations $(2-50 \mu \mathrm{g} / \mathrm{mL})$ for $16 \mathrm{~h}$. RNA of IFI35 and 18S rRNA were amplified quantitatively using a real-time PCR system and expression wasexamined. (B) Cell lysates were subjected to western blot analysis for IFI35 and actin after treatment with poly IC for $24 \mathrm{~h}$. (C) The cells were treated with $30 \mu \mathrm{g} / \mathrm{mL}$ poly IC for up to $24 \mathrm{~h}$. The expression of IFI35 mRNA was measured as in (A). (D) The cells were treated with poly IC as in (C), and western blot analyses for IFI35 and actin were performed as in (B). Bars represent the

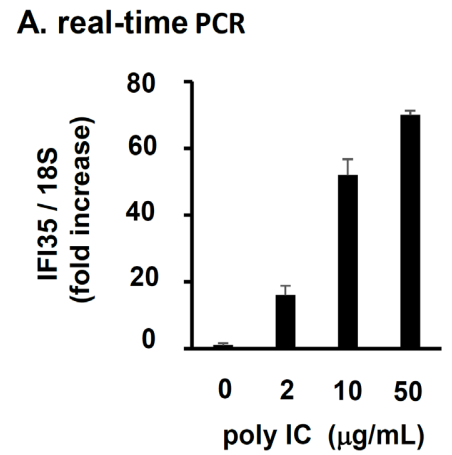

B. western blotting

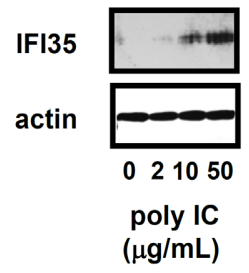

\section{C. real-time PCR}

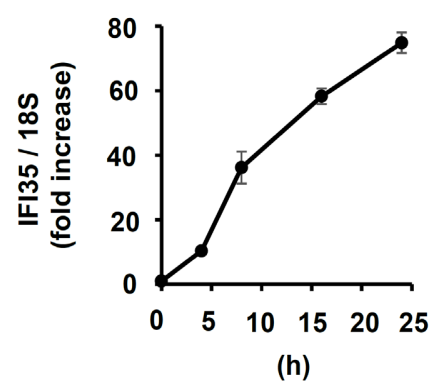

D. western blotting

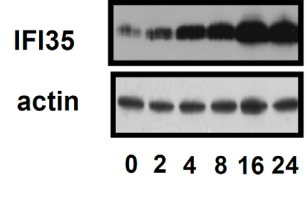

(h) means \pm SD $(n=3)$.

(Fig. 3B). Silencing IFI35 decreased the mRNA (Fig. 3A) and protein (Fig. 3C) expression of CCL5 and CXCL10, while it did not affect the mRNA expression of RIG-I or MDA5 (Fig. 3A).

\section{Discussion}

ISGs are reported to play regulatory functions to control innate immune reactions [12]. In the present study, we found that TLR3 activation by poly IC induces expression of IFI35 in cultured normal human MCs. Because IFI35 is an ISG and has a leucine zipper domain [12], we hypothesized that IFI35 may have a role in IFN-related innate immune reactions by regulating the expression of other ISGs. Since we found that CCL5 and CXCL10 are TLR3 activation-related representative proinflammatory chemokines in poly IC treated-MCs, we therefore examined the effect of RNA interference against IFI35 on the expression of CCL5 and CXCL10 $[10,11]$. Furthermore, we discovered that the IFN- $\beta$ synthesized de novo following TLR3 activation may be a key molecule in the regional inflammatory cascades in MCs in our experiment [6-11], and this IFN- $\beta$ is involved in the IFN- $\beta /$ RIG-I/CCL5 and IFN- $\beta$ /MDA5/CXCL10 signaling axes in cultured normal human MCs $[10,11]$.

In the present study, we examined the role of IFI35 in these axes using siRNA. We found that IFI35 positively regulated protein expression of RIG-I and MDA5, and both mRNA and protein of CCL5 and CXCL10 in normal human MCs treated with poly IC. However, IFI35 did not affect the protein expression of phosphorylation of STAT1, or the mRNA of RIG-I and MDA5. This suggests that IFI35 may, unexpectedly, enhance the expression of RIG-I and MDA5 proteins post-transcriptionally by increasing the translation or decreasing the degradation of RIG-I and MDA5 proteins. We found that IFI35 may upregulate the inflammatory reactions induced by viral infection or "pseudoviral immunity" in MCs, although this theory remains preliminary. Interestingly, our present findings seem to contradict the results of a previous study which showed that IFI35 negatively regulates RIG-I in HEK293 cells infected with 


\section{Kidney \\ Blood Pressure \\ Research}

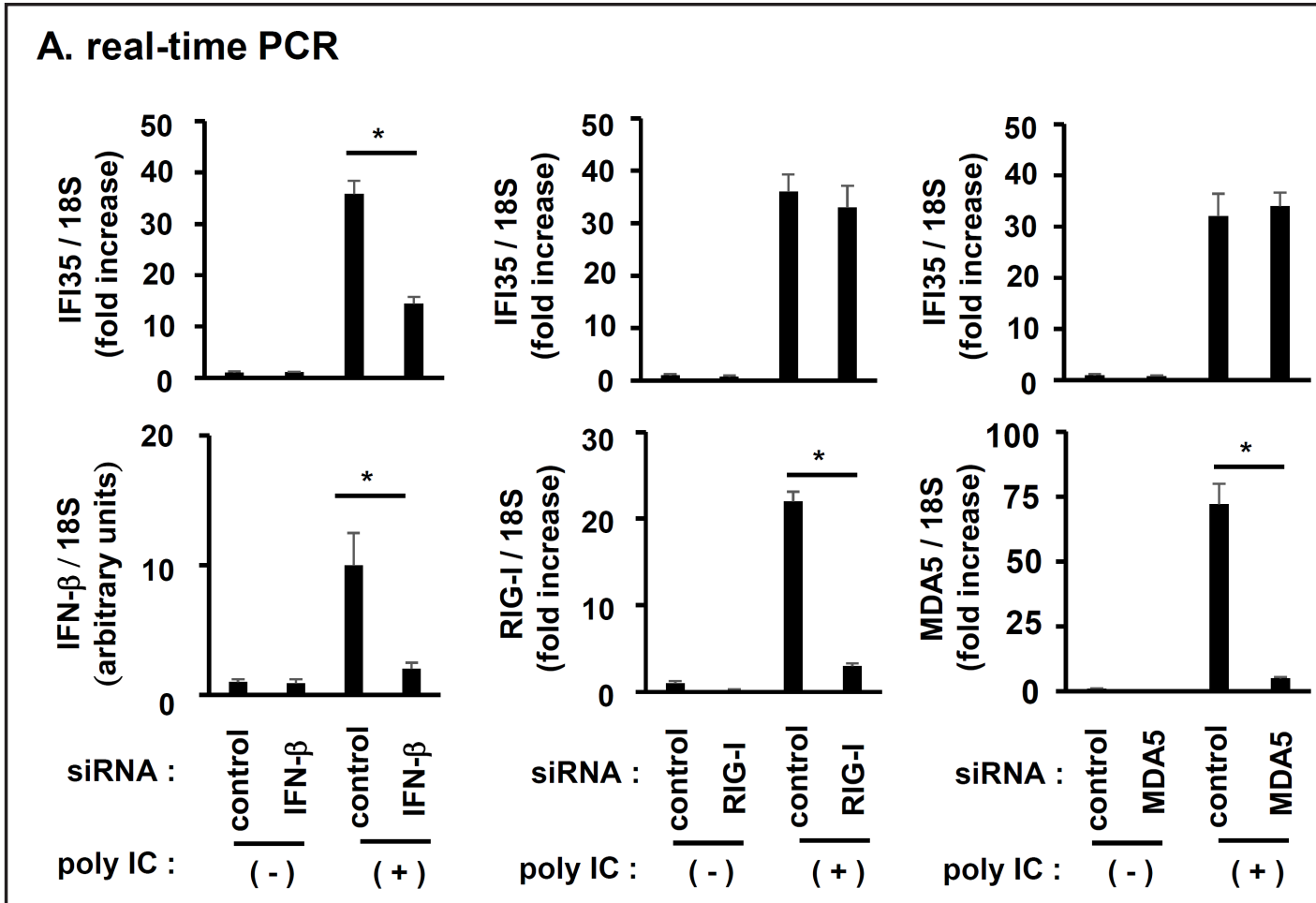

\section{B. western blotting}

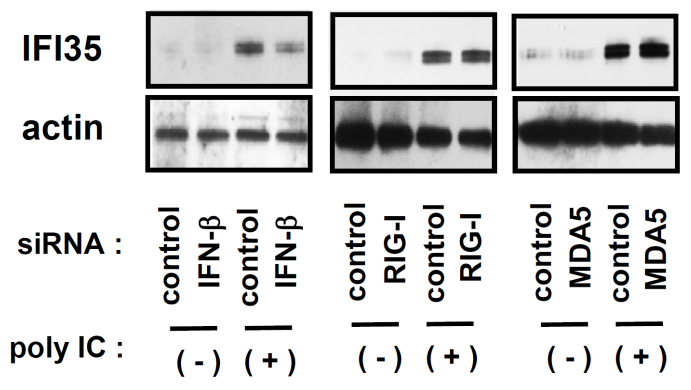

Fig. 2. IFN- $\beta$ is involved in IFI35 expression induced by poly IC. Cells were transfected with siRNA against IFN- $\beta$, RIG-I or MDA5. Subsequently, $30 \mu \mathrm{g} / \mathrm{mL}$ poly IC was added to the cultures. (A) The cells were subjected to real-time PCR analysis after additional incubation for $16 \mathrm{~h}$ (for mRNA of IFI35, RIG-I or MDA5) or 4 $\mathrm{h}$ (for mRNA of IFN- $\beta$ ) $\left(\mathrm{n}=3\right.$; $\left.{ }^{*} \mathrm{p}<0.01\right)$. (B) Cells were lysed after an additional $16 \mathrm{~h}$ incubation, and western blot analyses for IFI35 and actin were performed.

Sendai virus [13]. Although the reasons for this discrepancy are not known, the contradicting results may be due to the differences in cell type (primary normal human MCs versus immortalized cell line HEK293) or experimental conditions (addition of authentic doublestranded RNA poly IC versus infection with Sendai virus). IFI35 has a leucine zipper domain [12] and interacts with another ISG named N-myc interacting protein (Nmi) to form a 200$400 \mathrm{kDa}$ high molecular mass complex in response to IFN- $\beta$ treatment in HT29 colon cancer cells [15]. A protein-protein interaction was observed between IFI35 and RIG-I, which promotes degradation of RIG-I protein in HEK293 cells using an overexpression system 


\section{Kidney Blood Pressure Research}

Fig. 3. Effect of RNA interference against IFI35 on the expression of STAT1, phosphorylated STAT1 (P-STAT1), RIG-I, MDA5, CCL5, and CXCL10 induced by poly IC. The cells were transfected with siRNA against IFI35 and were incubated for 48 h. Then, the cells were treated with $30 \mu \mathrm{g} / \mathrm{mL}$ poly IC for $16 \mathrm{~h}$. (A) The levels of the indicated RNAs were analyzed using quantitative real-time RT-PCR $\quad(n=3$, ${ }^{*} \mathrm{p}<0.01$ ). (B) Cells were lysed and the indicated proteins were analyzed by western blotting. (C) Cell-conditioned medium was collected and the concentration of CCL5 and CXCL10 proteins in the medium were measured by ELISAs $(n=3, * p<0.01)$.

\section{A. real-time PCR}
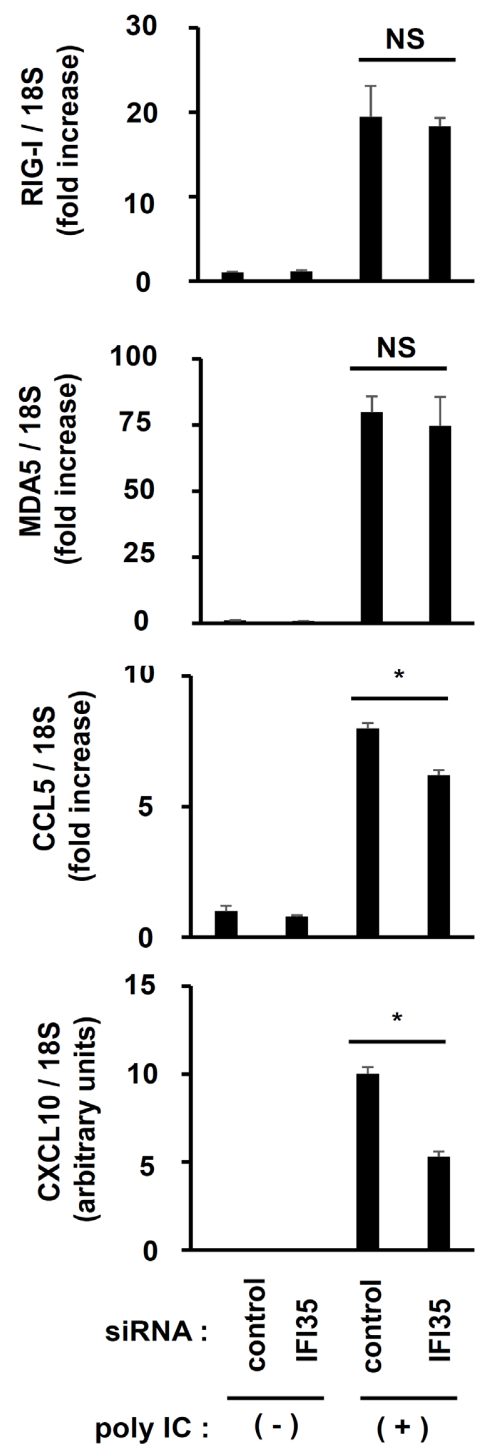

\section{B. western blotting}

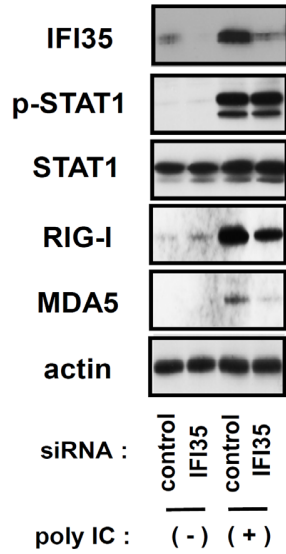

C. ELISA
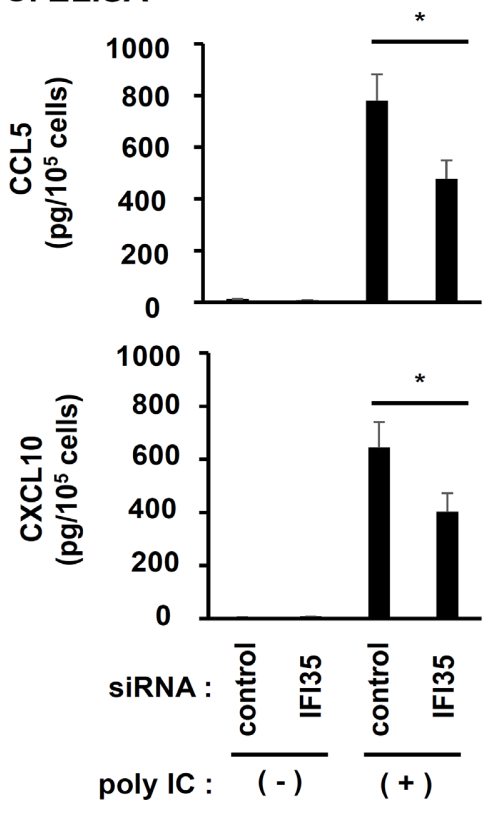

[13]. The mechanisms by which IFI35 increased the protein expression of RIG-I and MDA5 in the present study are not clear yet. However, the inconsistency between the present study and the previous study suggests that the mechanisms by which IFI35 modifies immune reactions may be complicated, and future studies should clarified the details of its biological characteristics.

Clinically, so far it has been reported that IFI35 is related with the pathogenesis of various human diseases including Diamond-Blackfan Anemia [16], foot-and-mouth disease virus infection [17], and atopic dermatitis [18]. These reports suggest that IFI35 may be involved in the pathogenesis of various inflammatory diseases related to viral infection and allergic reactions. However, there has been no direct evidence for the involvement of IFI35 in human inflammatory renal diseases, and this should be clarified in future clinical investigations. In this context, our present data may add another aspect of previously unknown IFI35 function in glomerular inflammation. We found that ISGs are involved glomerular inflammation triggered by TLR3 signaling [6-9]. Since ISGs and proinflammatory chemokines/cytokines 


\section{Kidney \\ Blood Pressure Research}

induced by activation of TLRs play protective or deleterious roles during the pathogenesis of glomerular inflammation in MCs $[1,3,4,6]$, we believe detailed understanding of interaction of these molecules in immune reactions may lead to the development of new therapeutic strategies for CKD.

\section{Conclusion}

We report that IFI35 positively regulates the TLR3-dependent expression of CCL5 and CXCL10, at least in part, by enhancing the expression of RIG-I and MDA5 proteins (Fig.4). IFI35 may positively regulate innate immune reactions in MCs, and may be involved in the pathogenesis of glomerular inflammation.

\section{Disclosure Statement}

The authors declare no conflict of interest.

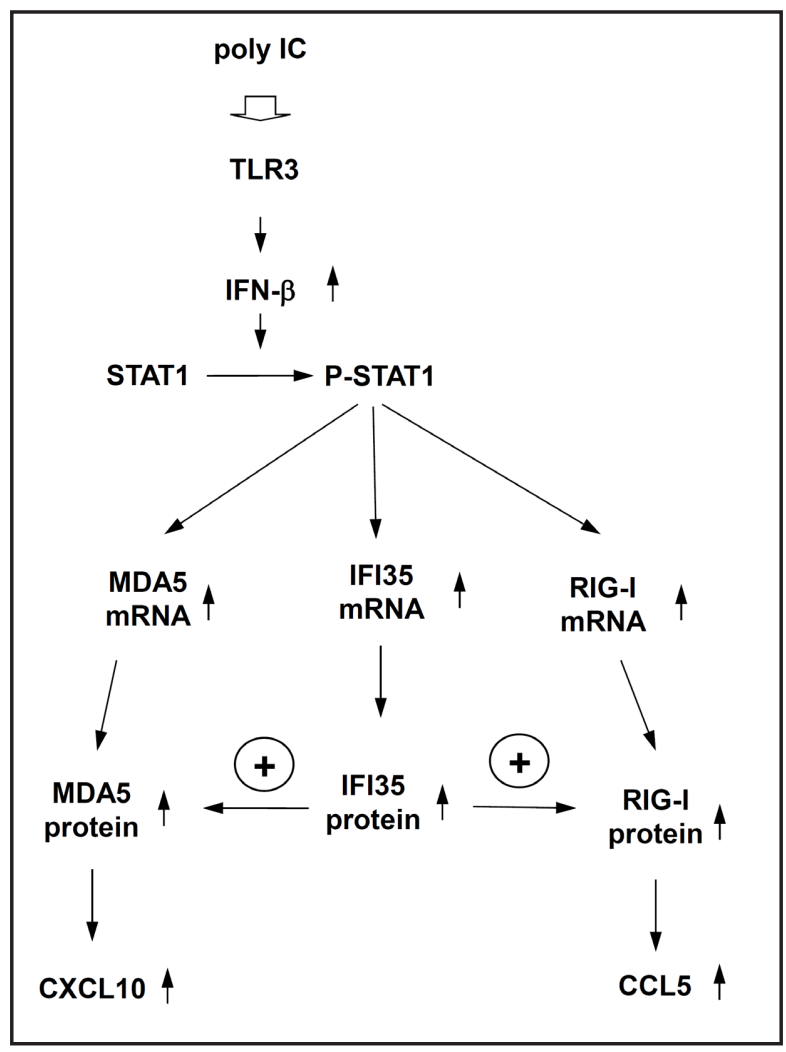

Fig. 4. Proposed role of IFI35 in enhancing the expression of RIG-I/CCL5 and MDA5/CXCL10 downstream of TLR3, IFN- $\beta$ and P-STAT1 in MCs.

\section{Acknowledgements}

This work was supported by grants-in-aid for Science from The Ministry of Education, Culture, Sports, Science and Technology of Japan (JSPS KAKENHI Grant Number 25461615 and 16K10055 to T. I. and H. T.), and a grant from Okinaka Memorial Lifestyle Related Disease Foundation (T. I.). The authors thank K. Nakata, M. Ono and K. Munakata for their help.

\section{References}

1 Robson MG: Toll-like receptors and renal disease. Nephron Exp Nephrol 2009;113:e1-e7.

2 Coppo R, Amore A, Peruzzi L, Vergano L, Camilla R: Innate immunity and IgA nephropathy. J Nephrol 2010;23:626-632.

3 Anders HS: Pseudoviral immunity - a novel concept for lupus. Trend Mol Med 2009;15:553-561.

4 Tanaka H, Imaizumi T: Inflammatory chemokine expression via toll-like receptor 3 signaling in normal human mesangial cells. Clin Dev Immunol 2013;2013:984708.

5 Migliorini A, Ebid R, Scherbaum CR, Anders HJ: The danger control concept in kidney disease: mesangial cells. J Nephrol 2013;26:437-449.

6 Imaizumi T, Shimada T, Matsumiya T, Yoshida H, Watanabe S, Tsuruga K, Kawaguchi S, Murakami K, Joh K, Tanaka H: Interferon-stimulated gene 15, a type I interferon-dependent transcript, is involved in a negative feedback loop in innate immune reactions in human mesangial cells. Nephron 2016;132:144-152. 


\section{Kidney \\ Blood Pressure Research}

7 Imaizumi T, Tanaka H, Mechti N, Matsumiya T, Yoshida H, Aizawa-Yashiro T, Tsuruga K, Hayakari R, Satoh K: Polyinosinic-polycytidylic acid induces the expression of interferon-stimulated gene 20 in mesangial cells. Nephron Exp Nephrol 2011;119:e40-e48.

8 Imaizumi T, Aizawa-Yashiro T, Matsumiya T, Yoshida H, Watanabe S, Tsuruga K, Tatsuta T, Xing F, Hayakari R, Meng P, Tanaka H: Interaction between interferon-stimulated gene 56 and melanoma differentiationassociated gene 5 in TLR3 signaling in normal human mesangial cells. Am J Nephrol 2013;37:118-125.

9 Watanabe S, Imaizumi T, Tsuruga K, Aizawa T, Ito T, Matsumiya T, Yoshida H, Joh K, Ito E, Tanaka H: Glomerular expression of myxovirus resistance protein 1 (Mx1) in human mesangial cells: possible activation of innate immunity in the pathogenesis of lupus nephritis. Nephrology (Carlton) 2013;18:833837.

10 Imaizumi T, Tanaka H, Matsumiya T, Yoshida H, Tanji K, Tsuruga K, Oki E, Aizawa-Yashiro T, Ito E, Satoh K: Retinoic acid-inducible gene-I is induced by double-stranded RNA and regulates the expression of CC chemokine ligand (CCL) 5 in human mesangial cells. Nephrol Dial Transplant 2010;25:3534-3539.

11 Imaizumi T, Aizawa-Yashiro T, Tsuruga K, Tanaka H, Matsumiya T, Yoshida H, Tatsuta T, Xing F, Hayakari $\mathrm{R}$, Satoh K: Melanoma differentiation-associated gene 5 regulates the expression of a chemokine CXCL10 in human mesangial cells: implications for chronic inflammatory renal diseases. Tohoku J Exp Med 2012;228:17-26.

12 Bange FC, Voge, U, Flohr T, Kiekenbeck M, Denecke B, Bottger EC: IFP35 is an interferon-induced leucine zipper protein that undergoes interferon-regulated cellular redistribution. J Biol Chem 1994;269:10911098.

13 Das A, Dinh PX, Panda D, Pattnaik AK: Interferon-inducibe protein IFI35 negatively regulates RIG-I antiviral signaling and supports vesicular stomatitis virus replication. J Virol 2014;88:3103-3113.

14 Imaizumi T, Aratani S, Nakajima T, Carlson M, Matsumiya T, Tanji K, Ookawa K, Yoshida H, Tsuchida S, McIntyre TM, Prescott SM, Zimmerman GA, Satoh K: Retinoic acid-inducible gene-I (RIG-I) is induced in endothelial cells by LPS and regulates expression of COX-2. Biochem Biophys Res Commun 2002;292:274279.

15 Zhou X, Liao J, Meyerdierks A, Feng L, Naumovski L, Bottger EC, Omary MB: Interferon- $\alpha$ induces nmiIFP35 heterodimetric complex formation that is affected by the phosphorylation of IFP35. J Biol Chem 2000;275:21364-21371.

16 Pesciotta EN, Lam H-S, Kossenkov A, Ge J, Showe LC, Mason PJ, Bessler M, Speicher DW: In-depth, labelfree analysis of the erythrocyte cytoplasmic proteome in Diamon Blackfan Anemia identifies a unique inflammatory signature. PLoS One 2015;10:e0140036.

17 Zheng W, Li X, Wang J, Li X, Cao H, Wang Y, Zeng Q Zheng SJ: A dritical role of interferon-induced protein IFP35 in the type I interferon response in cells induced by foot-and-mouth disease virus (FMDV) protein 2C. Arch Virol 2014;159:2925-2935.

18 Rebane A, Zimmermann M, Aab A, Baurecht H, Koreck A, Karelson M, Abram K, Metsalu T, Philap M, Meyer N, Folster-Holst R, Nagy N, Kemeny L, Kingo K, Vilo J, Illig T, Akdis M, Franke A, Novak N, Weidinger S, Akdis CA: Mechanisms of IFN- $\gamma$-induced apoptosis of human skin keratinocytes in patients with atopic dermatitis. J Allergy Clin Immunol 2012;129:1297-1306. 